\title{
New Small Scale Equipment for Obtaining Dill and Cumin Essential Oils
}

\author{
DENISA E. DUTA ${ }^{1 \#, ~ D A V I D ~ L . ~ C O M A N I C I U ~}{ }^{2}$, ALINA CULETU ${ }^{1 \#, ~ M I O A R A ~ N E G O I T A ~}{ }^{1 \#,}$ \\ VALENTIN IONESCU ${ }^{1 \#, ~ H A R A L D ~ B A R Z A N ~}{ }^{2}$, VALENTIN L. ORDODI ${ }^{2,3 *}$, GABRIELA BARZAN $^{2}$ \\ ${ }^{1}$ National Institute of Research and Development for Food Bioresources, IBA Bucharest, 6 Dinu Vintila, 021102, \\ Bucharest, Romania \\ ${ }^{2}$ SC Natural Ingredients R\&D SRL, 10/1 Tabacari Str., 505200, Fagaras, Romania \\ ${ }^{3}$ Politehnica University of Timisoara, 2 Victoriei Sq., 300006, Timisoara, Romania
}

Essential oils of natural origin are excellent raw materials for the food industry as well as the pharmaceutical and cosmetic industries. In this study, a new equipment, designed at small scale, which facilitates the yield of essential oils from various plant sources via hydrodistillation using the classical Clevenger apparatus is presented. The working of this equipment consists in oils distillation from raw plant material with steam, recirculating the water and collecting the volatile oil yield. The proposed designed equipment ensures high yield while keeping energy consumption to a minimum using proper thermal insulation. The yielded product is within the quality parameters for natural products imposed by regulators.

Keywords: volatile natural oils, hydrodistillation, Clevenger apparatus, dill, cumin

Essential oils can be isolated from plant material using classical, widespread procedures (azeotropic distillation, high-pressure extraction) or special procedures like supercritical fluid extraction. Steam distillation is a widely used technique in both laboratory and industrial equipments.

The entrainment of essential oils is a complex process. In the first stage, the oil diffuses from the cells of the plant material. The diffusion happens faster in green plants as opposed to dry plants. Furthermore, the oils are found in unequal ratios in the various anatomical compartments of the plant. The chemical composition of the oils can undergo changes during steam distillation due to collateral chemical reactions, such as isomerization, polymerization, transesterifications etc.

The usage of steam distillation is based on the fact that all components of an essential oil achieve high volatility at temperatures below their boiling point, causing them to be easily entrained by the steam. The steam forms azeotropic mixtures with the essential oils. The steam then condenses, resulting an organic phase (containing the essential oil) and an aqueous phase. The order in which the components of the oil are entrained primarily depends on their chemical polarity, and less so on their volatility. Thus, the first entrained components are primarily oxygenated terpenic compounds, followed by carbohydrates, which have lower boiling points but higher chemical polarity.

Carvone is a monoterpene, a ketone type, belonging to the class of terpenoids - a group of natural substances containing isoprene units in their chemical structure [1]. Carvone is a very important component of the fragrance and perfume industry [2]. Carvone can be obtained by extracting essential oils. Dill and cumin are sources of carvone. Dill is an annual plant, cultivated in subtropical or temperate regions. Cumin (Cuminum cyminum) is an annual flowering plant which grows spontaneously in Asia, Europe, northern Africa and the southwestern region of the US, and has been used as a spice probably since the ancient era [3].

Certain factors, including genotype, nutrient intake, and growth conditions are known to influence the carvone and limonene content of essential oils. Studies have shown that sunny dry weather during maturation will increase the carvone content, while cold wet weather may increase the terpenic hydrocarbon content, especially limonene $[4,5]$. Also, differences in chemical composition of essential oils were attributed to environmental conditions, geographic appurtenance, climatic, genetic, chemotypes, plant age, the soil purity, phase of vegetation, anatomical part of plant and harvesting season [6].

The aim of this study was to obtain essential oils from dill and cumin, both from seeds and grass, by steam distillation, as well as to characterize the oils by GC-MS and ${ }^{1} \mathrm{H}-\mathrm{NMR}$ techniques.

\section{Experimental part}

Materials and methods

A small scale equipment purposed for the extraction of essential oils via steam distillation inspired by the Clevenger apparatus was designed and built at the Natural Ingredients R\&D Ltd facility [7-10]. The design of the experimental equipment and its schematic are presented in Figure 1.

\footnotetext{
*email:valichem22@gmail.com
} 


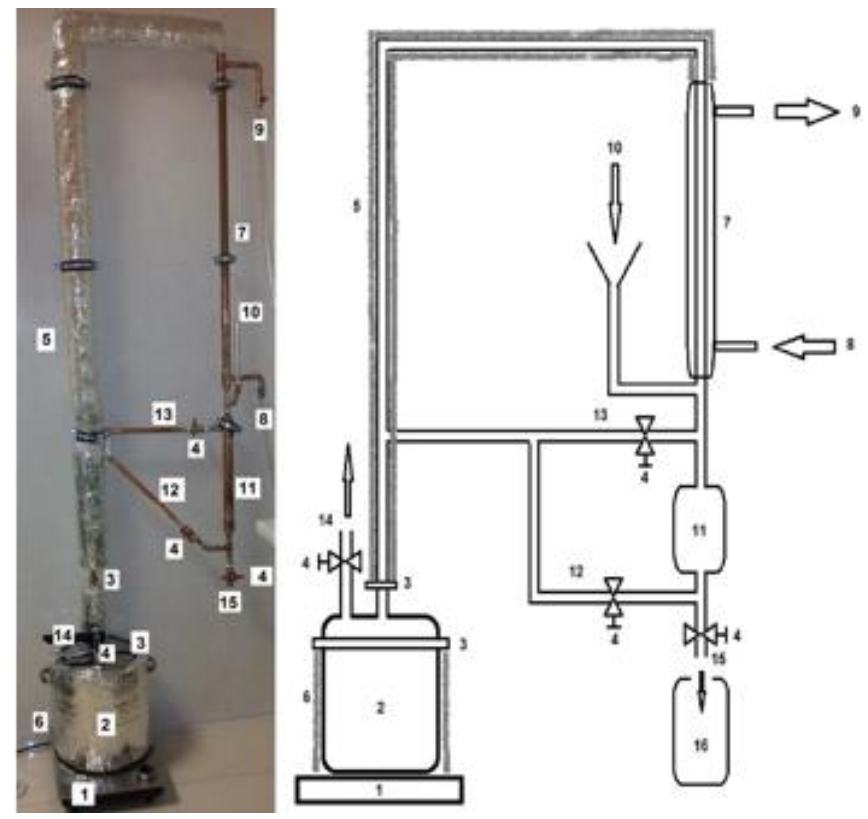

Fig. 1. Experimental small scale equipment for obtaining essential oils via steam distillation overview and design. Components: electric heater (1), cauldron (2), flange (3), taps (4), insulated steam duct (5), cauldron insulation (6), double-pipe heat exchanger (7), cooling inlet (8) and outlet (9), fill port (10), collector vessel (11), recirculation route $d_{\text {oil }}<\mathrm{dH}_{2} \mathrm{O}(12)$, recirculation route $d_{\text {oil }}>d_{\mathrm{H} 2 \mathrm{O}}$ (13), ventilation nozzle (14), drain nozzle (15) and drain vessel (16). The experimental equipment

The equipment is fitted with an industry-standard stainless steel cauldron (2) sporting a total volume of $\mathrm{V}_{\text {total }}=9 \mathrm{~L}$ and a fill volume $\mathrm{V}_{\text {fill }}=7 \mathrm{~L}$. The plant material is placed inside a wire netting on a metal holder $3 \mathrm{~cm}$ above the bottom of the cauldron, thus avoiding direct contact of the plant material with the hot surface of the bottom, which could lead to the alteration of the obtained essential oils. The cauldron is thermally insulated (6) with mineral wool. A tap allows the interior of the cauldron to communicate with the outside, but the tap is shut once the cauldron has been filled. The cauldron is heated up using a $1 \mathrm{~kW}$ electric heater (1). The resulting steam is led through a double pipe (or "pipe-inpipe') heat exchanger (7) through a mineral wool insulated copper duct with an internal diameter of $15 \mathrm{~mm}$. Steam condensation occurs at the heat exchanger, whose cooling is provided by the laboratory's water supply. The entrained oil then accumulated inside the $300 \mathrm{~mL}$ copper-made collector vessel (11) and separates from the aqueous phase (condensate) due to their immiscibility. The condensate is then led back to the cauldron via two routes, the choice of which depends on the density of the oil: one route (12) for oils with a density lower than water, and another route (13) for oils with higher density. Both routes are fitted with taps. After the steam distillation procedure is finished, the collector vessel (11) containing the essential oils and excess condensate is drained inside a recipient (16) through a drain tap (15).

\section{Obtaining cumin and dill seed essential oil}

Two kilograms of dill (Anethum graveolens L.) and cumin (Cuminum cyminum), acquired from a local producer (both seeds and grass of each species) were used to obtain the essential oils. They were split into batches according to Table 1 and fed into the extraction equipment.

Table 1

DISTRIBUTION OF THE DILL AND CUMIN SAMPLES

\begin{tabular}{|c|c|c|}
\hline \multicolumn{3}{|c|}{ Beeds (dill/cumin) } \\
\hline \multicolumn{3}{|c|}{ Batch mass (g) } \\
\hline Batch 1 & 700 & 6.3 \\
\hline Batch 2 & 700 & 6.3 \\
\hline Batch 3 & 600 & 6.4 \\
\hline \multicolumn{3}{|c|}{ Grass (dill/cumin) } \\
\hline Batch 1 & 400 & 6.6 \\
\hline Batch 2 & 400 & 6.6 \\
\hline Batch 3 & 400 & 6.6 \\
\hline Batch 4 & 400 & 6.6 \\
\hline Batch 5 & 400 & 6.6 \\
\hline
\end{tabular}

The steam distillation period for each batch was $12 \mathrm{~h}$. After the separation and weighing of the crude essential oil resulted from each batch, the batch-wise and global yields were computed for each essential oil type. 
GC-MS and ${ }^{1} H$-NMR analysis of the extracted essential oils

For the GC-MS analysis a gas chromatograph (Focus GC) coupled with an ion-trap mass spectrometer (POLARIS Q) (Thermo Fisher Scientific, US) was used. The gas chromatograph was fitted with a TriPlus autosampler (Thermo Fisher Scientific, US) and a SSL injector. Separation of the compounds from the essential oils was made using a TG5SILMS (60 mx, $0.25 \mathrm{~mm}$, film thickness $0.25 \mathrm{pm}$; $5 \%$ phenyl methylpolysiloxane) (Thermo Fisher Scientific, US) capillary column with helium as carrier gas, at a constant flow of $1 \mathrm{~mL} / \mathrm{min}$. Sample analysis was conducted at $70 \mathrm{eV}$ with positive electron impact ionization $\left(\mathrm{EI}^{+}\right)$in full-scan mode, with a mass range $\mathrm{m} / \mathrm{z}=40-350$. Injector and transfer line temperatures were set at 250 and $300{ }^{\circ} \mathrm{C}$, respectively. $\mathrm{GC}$ oven temperature was set at $40{ }^{\circ} \mathrm{C}$ for the first $2 \mathrm{~min}$, then linearly heated up to $280^{\circ} \mathrm{C}$ with a rate of $5^{\circ} \mathrm{C} / \mathrm{min}$, and maintained for $10 \mathrm{~min} .1 \mu \mathrm{L}$ of each oil sample (diluted $1 / 10,1 / 100,1 / 200,1 / 600,1 / 5000,1 / 5500$ in hexane, v/v) was injected (split injection, 1:100 ratio). The data was acquired and processed using the Xcalibur software package.

For ${ }^{1} \mathrm{H}-\mathrm{NMR}$ analysis, a Bruker Ascend $400 \mathrm{MHz}$ spectrometer was used with the following parameter: $45^{\circ}$ pulse angle, no power attenuation, acquisition time $2.05 \mathrm{sec}, 6.4 \mathrm{KHz}$ spectral window, 16 scans, 26K sampled points with delay $\mathrm{d} 1=1 \mathrm{~s}$. Free induction delay (FID) was not processed before the Fourier transform. The average acquisition duration of a ${ }^{1} \mathrm{H}-\mathrm{NMR}$ spectrum was $\sim 2 \mathrm{~min}$. The spectra were acquired after diluting the samples with deuterated chloroform $\left(\mathrm{CDCl}_{3}\right)$ at a $2: 8 \mathrm{v} / \mathrm{v}$ ratio.

\section{Results and discussions}

Table 2 shows the entrainment yields for each batch and processed plant, as well as the average yield for each resulting product.

Table 2

DATA ABOUT THE EXTRACTION PROCESS

\begin{tabular}{|c|c|c|c|c|c|}
\hline Batch no. & $\begin{array}{l}\text { Plant mass } \\
(\mathrm{g})\end{array}$ & $\begin{array}{r}\text { Extracted } \\
\text { volatile oil }(g)\end{array}$ & Yield (\%) & $\begin{array}{l}\text { Total extracted } \\
\text { volatile oil }(\mathrm{g})\end{array}$ & $\begin{array}{l}\text { Average } \\
\text { yield }(\%)\end{array}$ \\
\hline \multicolumn{6}{|c|}{ Dill seed } \\
\hline Batch 1 & 700 & 20.3 & 2.9 & \multirow{3}{*}{59.3} & \multirow{3}{*}{2.96} \\
\hline Batch 2 & 700 & 19.8 & 2.8 & & \\
\hline Batch 3 & 600 & 19.2 & 3.2 & & \\
\hline \multicolumn{6}{|c|}{ Dill grass } \\
\hline Batch 1 & 400 & 3.2 & 0.8 & \multirow{5}{*}{16.1} & \multirow{5}{*}{0.805} \\
\hline Batch 2 & 400 & 3.3 & 0.82 & & \\
\hline Batch 3 & 400 & 3.2 & 0.8 & & \\
\hline Batch 4 & 400 & 3.1 & 0.77 & & \\
\hline Batch 5 & 400 & 3.3 & 0.82 & & \\
\hline \multicolumn{6}{|c|}{ Cumin seed } \\
\hline Batch 1 & 700 & 37.1 & 5.3 & \multirow{3}{*}{108} & \multirow{3}{*}{5.4} \\
\hline Batch 2 & 700 & 38.5 & 5.5 & & \\
\hline Batch 3 & 600 & 32.4 & 5.4 & & \\
\hline \multicolumn{6}{|c|}{ Cumin grass } \\
\hline Batch 1 & 400 & 2.9 & 0.72 & \multirow{5}{*}{14.6} & \multirow{5}{*}{0.73} \\
\hline Batch 2 & 400 & 3 & 0.75 & & \\
\hline Batch 3 & 400 & 2.9 & 0.72 & & \\
\hline Batch 4 & 400 & 2.9 & 0.72 & & \\
\hline Batch 5 & 400 & 2.9 & 0.72 & & \\
\hline
\end{tabular}

The extracted dill seed oil is a pale yellow liquid. Its smell is cumin-like, fresh, neat and spicy. Its taste is warm, lightly spicy, with an intense sweet-aromatic flavor. Its relative density was 0.9274 and its refractive index $n_{D}{ }^{20}$ was 1.5178 .

The extracted cumin seed oil is a pale yellow liquid with a penetrating smell and a warm, spicy taste. Its relative density was 0.9201 and its refractive index $n_{D}^{20}$ was 1.4933 .

The peaks from the chromatogram of each extract were identified by comparing their retention times to reference standards. Identification of the constituting chemical compounds was done by analyzing GC retention times, and the confirmation and interpretation of mass spectra was done using the NIST Mass Spectral Library provided by the GCMS software and data from other studies. Quantitative data was expressed as non-corrected percentage of area. Each probe (and its dilutions) was injected thrice. The relative standard deviations for the major components did not exceed $2 \%$. Tables 3 and 4 show the composition of the oils extracted from dill and cumin, respectively.

Figure 2 shows the chromatograms for the dill seed and grass essential oils compared to a reference dill seed oil from Sigma. 
Table 3

EXTRACTED DILL OIL COMPOSITION

\begin{tabular}{|c|c|c|c|c|c|c|c|c|}
\hline \multirow[t]{2}{*}{ No. } & \multirow[t]{2}{*}{ Component name } & \multirow{2}{*}{$\begin{array}{l}\text { RT, } \\
\text { min. }\end{array}$} & \multirow{2}{*}{$\begin{array}{c}\text { Dill seed oil } \\
\text { standard, } \\
\%\end{array}$} & \multicolumn{2}{|c|}{ Obtained oils } & \multicolumn{3}{|c|}{ Identifiers } \\
\hline & & & & $\begin{array}{c}\text { Dill seed } \\
\text { oil, } \\
\%\end{array}$ & $\begin{array}{c}\text { Dill grass } \\
\text { oil, } \\
\%\end{array}$ & $\begin{array}{l}\text { CAS } \\
\text { No. }\end{array}$ & $\begin{array}{l}\text { MW, } \\
\text { g/mol }\end{array}$ & $\begin{array}{c}\text { Molecular } \\
\text { formula }\end{array}$ \\
\hline 1 & alpha-Pinene & 15.06 & 0.16 & 0.05 & 1.20 & $\begin{array}{c}7785- \\
70-8 \\
\end{array}$ & 136 & $\mathrm{C}_{10} \mathrm{H}_{16}$ \\
\hline 2 & $\begin{array}{l}\text { Bicyclo[3.1.1]hept-2-ene, } \\
\text { 2, 6-dimethyl-6-(4- } \\
\text { methyl-3-pentenyl)- }\end{array}$ & 16.78 & & 0.06 & 0.23 & $\begin{array}{c}2437- \\
95-8\end{array}$ & 136 & $\mathrm{C}_{10} \mathrm{H}_{16}$ \\
\hline 3 & $\alpha$-Phellendrene & 17.42 & 18.89 & 0.32 & 26.81 & $\begin{array}{c}99-83- \\
2\end{array}$ & 136 & $\mathrm{C}_{10} \mathrm{H}_{16}$ \\
\hline 4 & o-cymene & 18.08 & 17.91 & & 2.01 & $\begin{array}{l}527- \\
84-4\end{array}$ & 134 & $\mathrm{C}_{10} \mathrm{H}_{14} \mathrm{O}$ \\
\hline 5 & alfa-limonene & 18.25 & 32.94 & 34.03 & 23.42 & $\begin{array}{l}138- \\
86-3\end{array}$ & 136 & $\mathrm{C}_{10} \mathrm{H}_{16}$ \\
\hline 6 & L-Fenchone & 20.33 & 2.48 & & & $\begin{array}{l}126- \\
21-6\end{array}$ & 152 & $\mathrm{C}_{10} \mathrm{H}_{16} \mathrm{O}$ \\
\hline 7 & Trans-3(10)-Caren 2-ol & 23.24 & 2.03 & & 8.68 & & 152 & $\mathrm{C}_{10} \mathrm{H}_{16} \mathrm{O}$ \\
\hline 8 & Estragole & 23.53 & & 0.32 & 1.32 & $\begin{array}{l}140- \\
67-0\end{array}$ & 148 & $\mathrm{C}_{10} \mathrm{H}_{16} \mathrm{O}$ \\
\hline 9 & trans-dihydrocarvona & 23.78 & & 1.34 & 4.33 & $\begin{array}{l}5948- \\
04-09\end{array}$ & 152 & $\mathrm{C}_{10} \mathrm{H}_{12} \mathrm{O}$ \\
\hline 10 & Carvol/1-Carvone & 24.92 & 25.59 & 31.02 & 32.00 & $\begin{array}{c}99-49- \\
0 \\
\end{array}$ & 150 & $\mathrm{C}_{10} \mathrm{H}_{14} \mathrm{O}$ \\
\hline 11 & Anethole & 26.06 & & & & $\begin{array}{l}104- \\
46-1\end{array}$ & 148 & $\mathrm{C}_{10} \mathrm{H}_{12}$ \\
\hline 12 & $\begin{array}{c}\mathrm{o}- \\
\text { tymol/isothymol/carvacrol }\end{array}$ & 26.26 & & & & $\begin{array}{l}499- \\
75-2\end{array}$ & 150 & $\mathrm{C}_{10} \mathrm{H}_{14} \mathrm{O}$ \\
\hline 13 & Myristicin & 32.2 & & & & $\begin{array}{l}607- \\
91-0\end{array}$ & 192 & $\mathrm{C}_{10} \mathrm{H}_{14} \mathrm{O}$ \\
\hline 14 & Apiol & 34.56 & & 32.85 & & $\begin{array}{l}523- \\
80-8\end{array}$ & 222 & $\mathrm{C}_{12} \mathrm{H}_{14} \mathrm{O}_{4}$ \\
\hline 15 & Benzyl Benzoate/ascabiol & 38.01 & & & & $\begin{array}{l}120- \\
51-4\end{array}$ & 212 & $\mathrm{C}_{12} \mathrm{H}_{12} \mathrm{O}_{2}$ \\
\hline & & & 100 & 100.00 & 100.00 & & & \\
\hline
\end{tabular}

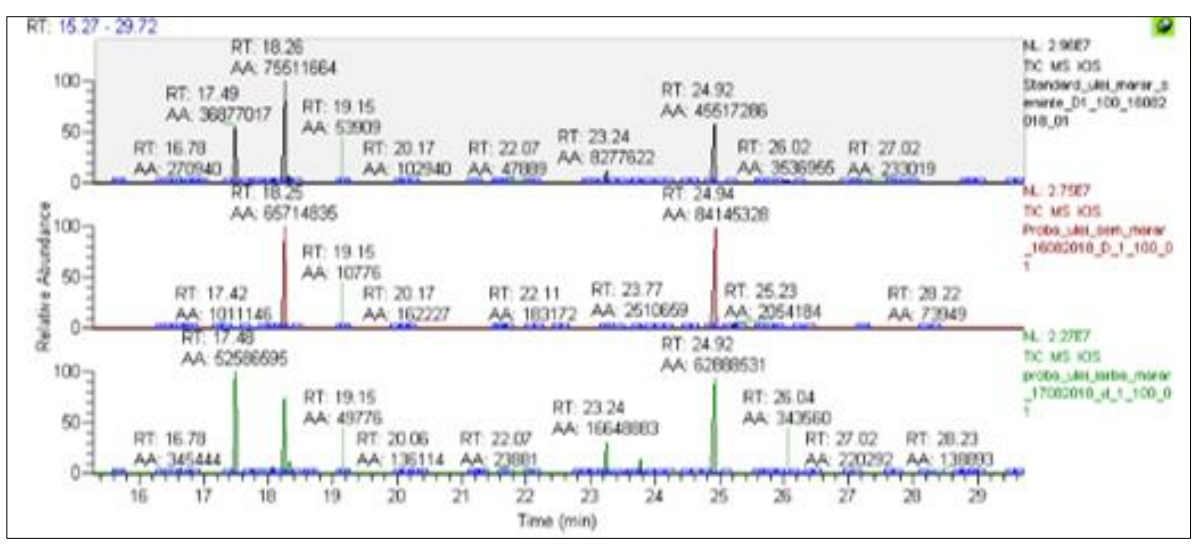

Fig. 2. Dill oil chromatograms. Top to bottom: reference dill seed oil (SIGMA), extracted dill seed oil, extracted dill grass oil

Table 4

EXTRACTED CUMIN OIL COMPOSITION

\begin{tabular}{|c|c|c|c|c|c|c|c|c|}
\hline \multirow[t]{2}{*}{ No. } & \multirow[t]{2}{*}{ Component name } & \multirow{2}{*}{$\begin{array}{l}\text { RT, } \\
\text { min. }\end{array}$} & \multirow{2}{*}{$\begin{array}{l}\text { Dill seed } \\
\text { oil } \\
\text { standard } \\
\%\end{array}$} & \multicolumn{2}{|l|}{ Obtained oils } & \multicolumn{3}{|l|}{ Identifiers } \\
\hline & & & & $\begin{array}{l}\text { Cumin seed } \\
\text { oil \% }\end{array}$ & $\begin{array}{l}\text { Cumin } \\
\text { grass oil \% }\end{array}$ & CAS No. & $\begin{array}{l}\text { MW, } \\
\text { g/mol }\end{array}$ & $\begin{array}{l}\text { Molecular } \\
\text { formula }\end{array}$ \\
\hline 1 & alpha-Pinene & 15.06 & 0.79 & 1.31 & & $7785-70-8$ & 136 & $\mathrm{C}_{10} \mathrm{H}_{16}$ \\
\hline 2 & $\begin{array}{l}\alpha, \alpha- \\
\text { Dihydroacetopheno } \\
\text { ne/ Ethanone }\end{array}$ & 15.97 & & & 0.10 & $1075-06-5$ & 152 & $\mathrm{C}_{8} \mathrm{H}_{8} \mathrm{O}_{3}$ \\
\hline 3 & 3-carene & 16.6 & 13.50 & 10.25 & & $13466-78-9$ & 136 & $\mathrm{C}_{10} \mathrm{H}_{16}$ \\
\hline
\end{tabular}




\begin{tabular}{|c|c|c|c|c|c|c|c|c|}
\hline 4 & $\begin{array}{l}\text { Bicyclo[3.1.1]hept- } \\
\text { 2-ene, 2, 6- } \\
\text { dimethyl-6-(4- } \\
\text { methyl-3- } \\
\text { pentenyl)- }\end{array}$ & 16.79 & & & 0.11 & $2437-95-8$ & 136 & $\mathrm{C}_{10} \mathrm{H}_{16}$ \\
\hline 5 & $\alpha$-Phellendrene & 17.47 & 1.07 & & & $99-83-2$ & 136 & $\mathrm{C}_{10} \mathrm{H}_{16}$ \\
\hline 6 & $\gamma$-terpinene & 17.84 & 0.18 & & & $99-85-4$ & 136 & $\mathrm{C}_{10} \mathrm{H}_{16}$ \\
\hline 7 & o-cymene & 18.06 & 10.11 & 21.60 & & $527-84-4$ & 134 & $\mathrm{C}_{10} \mathrm{H}_{14} \mathrm{O}$ \\
\hline 8 & alpha-limonene & 18.24 & 0.38 & 1.10 & 33.33 & $138-86-3$ & 136 & $\mathrm{C}_{10} \mathrm{H}_{16}$ \\
\hline 9 & $\delta$-terpinene & 19.16 & 26.19 & 14.52 & & $99-85-4$ & 136 & $\mathrm{C}_{10} \mathrm{H}_{16}$ \\
\hline 10 & Terpinolene & 20.06 & & 0.20 & & $586-62-9$ & 136 & $\mathrm{C}_{10} \mathrm{H}_{16}$ \\
\hline 11 & $\begin{array}{l}\text { Dehydro-p- } \\
\text { cymene/ } \alpha, 4- \\
\text { dimethylstyrene }\end{array}$ & 20.17 & & 0.54 & & $1195-32-0$ & 132 & $\mathrm{C}_{10} \mathrm{H}_{12} \mathrm{O}$ \\
\hline 12 & $\begin{array}{l}\text { trans-p-Mentha- } \\
\text { 2,8-dienol }\end{array}$ & 21.21 & & & 0.13 & & 152 & $\mathrm{C}_{10} \mathrm{H}_{16} \mathrm{O}$ \\
\hline 13 & $\begin{array}{l}\text { cis-p-Mentha-2,8- } \\
\text { dienol }\end{array}$ & 21.66 & & & 0.18 & $22771-44-4$ & 152 & $\mathrm{C}_{10} \mathrm{H}_{16} \mathrm{O}$ \\
\hline 14 & $\begin{array}{l}\text { Terpinenal-4/ para- } \\
\text { menthen-4-al }\end{array}$ & 23.08 & 0.20 & & & $2562-74-3$ & 154 & $\mathrm{C}_{10} \mathrm{H}_{18} \mathrm{O}$ \\
\hline 15 & Estragole & 23.46 & 1.16 & 0.79 & 0.32 & $140-67-0$ & 148 & $\mathrm{C}_{10} \mathrm{H}_{12} \mathrm{O}$ \\
\hline 16 & $\begin{array}{l}\text { trans- } \\
\text { dihydrocarvone }\end{array}$ & 23.78 & & & 0.12 & 1478602 & 152 & $\mathrm{C}_{10} \mathrm{H}_{12} \mathrm{O}$ \\
\hline 17 & trans-2-Caren-4-ol & 24.11 & & & 0.36 & $4017-82-7$ & 152 & $\mathrm{C}_{10} \mathrm{H}_{16} \mathrm{O}$ \\
\hline 18 & cis-Carveol & 24.74 & & & 0.22 & $1197-06-4$ & 152 & $\mathrm{C}_{10} \mathrm{H}_{16} \mathrm{O}$ \\
\hline 19 & Carvol/1-Carvone & 24.92 & & 42.01 & 64.64 & $99-49-0$ & 150 & $\mathrm{C}_{10} \mathrm{H}_{14} \mathrm{O}$ \\
\hline 20 & Cinnamaldehyde & 25.92 & & & 0.24 & $104-55-2$ & 132 & $\mathrm{C}_{9} \mathrm{H}_{8} \mathrm{O}$ \\
\hline 21 & Anethole & 26.02 & 2.23 & & 0.19 & $104-46-1$ & 148 & $\mathrm{C}_{10} \mathrm{H}_{12}$ \\
\hline 22 & 2-caren-10-al & 26.20 & 25.74 & 6.10 & & & 150 & $\mathrm{C}_{10} \mathrm{H}_{14} \mathrm{O}$ \\
\hline 23 & Carbicol & 26.27 & 16.84 & & & $63-54-9$ & 136 & $\mathrm{C}_{9} \mathrm{H}_{12} \mathrm{O}$ \\
\hline 24 & $\begin{array}{l}\text { p-mentha-1,4-dien- } \\
\text { 7-al }\end{array}$ & 27.22 & 0.49 & & & $22539-72-6$ & 152 & $\mathrm{C}_{10} \mathrm{H}_{16} \mathrm{O}$ \\
\hline 25 & $\begin{array}{l}\text { trans- } \\
\text { Caryophyllene }\end{array}$ & 29.97 & & 0.23 & & $87-44-5$ & 204 & $\mathrm{C}_{15} \mathrm{H}_{24}$ \\
\hline 26 & di-epi- $\alpha$-cedrene & 31.35 & 0.79 & & & & 204 & $\mathrm{C}_{15} \mathrm{H}_{24}$ \\
\hline 27 & $\delta$-Himachalene & 32.05 & & 0.11 & & & 204 & $\mathrm{C}_{15} \mathrm{H}_{24}$ \\
\hline \multirow[t]{2}{*}{28} & Apiol & 34.52 & 0.33 & & 0.08 & $523-80-8$ & 222 & $\mathrm{C}_{12} \mathrm{H}_{14} \mathrm{O}_{4}$ \\
\hline & & & 100 & 100.00 & 100.00 & & & \\
\hline
\end{tabular}

From the ${ }^{1} \mathrm{H}-\mathrm{NMR}$ analysis, the content of alpha-phellandrene, carvone and limonene from the extracted essential oils and from a reference dill grass essential oil (SIGMA) were determined. Table 5 presents the experimental results.

Table 5

THE MAJOR COMPOUNDS IN OILS BASED ON ${ }^{1} \mathrm{H}-\mathrm{NMR}$ ANALYSIS

\begin{tabular}{|c|c|c|c|c|}
\hline No. & Essential oil type & Alpha-phellandrene content (\%) & $\begin{array}{c}\text { Carvone content } \\
(\boldsymbol{\%})\end{array}$ & $\begin{array}{c}\text { Limonene content } \\
(\boldsymbol{\%})\end{array}$ \\
\hline 1 & Dill grass oil - Sigma & 18.8 & 25.0 & 32.0 \\
\hline 2 & Dill seed essential oil & - & 31.0 & 34.2 \\
\hline 3 & Dill grass essential oil & 27.0 & 32.0 & 24.2 \\
\hline 4 & Cumin seed essential oil & - & 64.0 & 1.5 \\
\hline 5 & Cumin grass essential oil & - & 42.0 & 33.2 \\
\hline
\end{tabular}

The experimental data showed that the highest carvone content was in the essential oil obtained by processing of cumin seeds. Cumin seed oil extraction also has the highest steam distillation yield, making them the best source of natural carvone in advantageous conditions.

\section{Conclusions}

The presented steam distillation equipment is very well suited for the extraction of essential oils from various raw plant materials. Due to the design of the equipment, contact of the plant material with the overheated bottom side of the cauldron is avoided, thus the essential oil yield will be of superior quality, as far as both physicochemical and 
organoleptic properties are concerned. The equipment is amenable to be built at semi-industrial scale to increase the production efficiency of these oils.

Acknowledgments:This work was supported by a grant of the Romanian National Authority for Scientific Research, contract no. 57/2016 (EXPERTAL), project type D no. 14/2017.

\section{References}

1.DE CARVALHO, C.C.C.R., DA FONSECA, M.M.R., Food Chem., 95, 2006, p. 413.

2.RĂDOIAȘ, G., BOSILCOV, A., BÂTIU, I., Odorante naturale în parfumeria modernă, Casa Carții de Știință, Cluj Napoca, 2013.

3.JOHRI, R.K., Pharmacogn. Rev., 5, no. 9, 2011, p. 63.

4.CHARLES, D.J., Caraway Antioxidant Properties of Spices, In Herbs and Other Sources, Springer, New York, 2012, p. 199.

5.SOLBERG, S.O., GORANSSON, M., PETERSEN, M.A., YNDGAARD, F., JEPPSON, S., 66, 2016, p. 351.

6.BERECHET, M.D., MANAILA, E., STELESCU, M.D., CRACIUN, G., Rev. Chim., 68, no. 12, 2017, p. 2787.

7.ACHIMESCU, N. \& all, Tehnologia fabricarii si repararii utilajului tehnologic, 2, Institutul Politehnic "T. Vuia” Timișoara, 1989.

8.PODE, V, Procese hidrodinamice, Ed. Politehnica, Timișoara, 2001.

9.GABOR, L., GABOR, D., Transfer termic în ingineria chimica, Ed. Politehnica, Timişoara, 2002.

10.CRAVEIRO, A.A., MATOS, F.J.A., de ALENCAR, J.W., J. Chem. Educ., 53, no. 10, 1976, p. 652.

$\overline{\text { Manuscript received: } 23.05 .2019}$ 